\title{
A red eye induced by a spontaneous carotid cavernous fistula
}

\author{
Yu Xia* \\ Department of Ophthalmology, Beijing Aerospace General Hospital, China
}

\begin{abstract}
A 73-year-old woman initially presented to our ophthalmology department with complaint of a red eye who was misdiagnosed with conjunctivitis during 2 months. Digital subtraction angiography (DSA) imaging revealed a carotid cavernous fistula (CCF). The purpose of this article is to describe ophthalmological complaints of CCFs to help ophthalmologist in understanding the clinical features of spontaneous CCF and avoid misdiagnosis.
\end{abstract}

\section{Case report}

Carotid cavernous sinus fistula (CCF) is a rare disorder that arises due to an abnormal communication between the cavernous sinus and the carotid arterial system, which may be divided into spontaneous or traumatic in relation to cause [1-2]. Spontaneous fistulas usually have low rates of arterial blood flow and result in less severe symptoms, with insidious onset, mild orbital congestion, proptosis and low or no bruit [3]. Many patients with spontaneous CCF may initially present to an ophthalmologist for any associated visual impairment and need to be identified. When the patient simply has a red eye, perhaps with minimal eyelid swelling, it may be believed that he or she has a chronic conjunctivitis [4]. Because the symptoms and signs of a spontaneous CCF often are mild, usually developing rather slowly, this lesion is often misdiagnosed initially [2]. The purpose of this article is to report an old woman with carotid cavernous sinus fistula who was misdiagnosed with conjunctivitis over 2 months, and help in understanding the clinical features of spontaneous CCF in order to prompt diagnostic studies.

A 73-year-old woman presented with complaints of right eye redness over 2 months. Two months prior to the clinic, she had been diagnosed as a presumed infectious conjunctivitis and an ophthalmologist prescribed topical antibiotics and lubricating eye drops for presumed conjunctivitis, but symptoms and signs did not improve. Ophthalmic examination showed best corrected visual acuity of 20/50 in the right and 30/50 in the left eye. The intraocular pressure was $28 \mathrm{~mm} \mathrm{Hg}$ in the right eye and $17 \mathrm{~mm} \mathrm{Hg}$ in the left eye, respectively, measured with a noncontact air puff tonometer. Slit lamp biomicroscopy revealed tortuous dilated conjunctival vessels and chemosis in the right eye. Pupils were equal in size and reacted normally to light without a relative afferent pupillary defect. Dilated fundus examination revealed slightly dilated vessels and mild vascular tortuosity in the right eye. Exophthalmometry showed $2 \mathrm{~mm}$ of right eye proptosis. Eye movements demonstrated mild limitation of abduction of the right eye. One month later she returned to the emergency department with a painful, swollen right eye and an intraocular pressure of $39 \mathrm{~mm} \mathrm{Hg}$ and limitation of her extraocular muscles in nearly all fields of gaze. Computed tomography scan (CT scan) showed an enlarged superior ophthalmic vein (SOV), and cerebral angiography showed fistulas between the meningeal branches of both the internal carotid arteries and the cavernous sinus.
Based on history and imaging studies, diagnosis of CCF was made. After the neurosurgical intervention, patient's symptoms resolved, conjunctival injection decreased, IOP returned to normal, proptosis decreased, and limitation of her extraocular muscles in nearly all fields of gaze had markedly improved.

\section{Discussion}

Spontaneous fistulas may present quite variably or insidiously in onset and generally tend to have less severe and acute presentations which are often initially misdiagnosed as other conditions, including thyroid eye disease, orbital inflammatory syndrome, or chronic conjunctivitis [5]. Accurate and early diagnosis is of utmost importance because treatment delay may lead to blindness or permanent neurologic deficits [6]. As shown by this case, this 73-year-old woman with spontaneous fistulas initially presented to an ophthalmologist with red eye was misdiagnosed as conjunctivitis, but she had experienced no benefit from topical antibiotic therapy. She subsequently developed double vision, conjunctival chemosis and increased intraocular pressure in the affected eye. In CCF, blood passes from the high-pressure arterial system to the low-pressure venous drainage system, and venous congestion may cause arterialization of conjunctival veins, chemosis, proptosis, diplopia with ophthalmoparesis, elevated intraocular pressure, and decreased visual acuity [5]. Therefore, we assumed that the ocular symptoms and signs in this 73 -year-old patient including elevated intraocular pressure, dilated and tortuous conjunctival vessels, chemosis, proptosis, diplopia and a dilated superior ophthalmic vein may be based on the venous congestion, and led us to strongly suspect a carotid-cavernous fistula (CCF) despite the absence of this finding on cerebral angiography. This patient underwent cerebral angiography, which confirmed a CCF characterized by an abnormal communication between the cavernous sinus and the internal carotid arteries. This case

${ }^{\star}$ Correspondence to: Yu Xia, department of Ophthalmology, Beijing Aerospace General Hospital, 7 Wanyuan North Road, Fengtai District, Beijing 100076, China

Key words: carotid cavernous sinus fistula, eye redness, conjunctival chemosis, proptosis, diplopia

Received: July 13, 2018; Accepted: July 20, 2018; Published: July 24, 2018 
illustrates the ocular manifestations that can be seen in the patients with spontaneous CCF. When treating complaints of eye redness with development of painful swelling, and proptosis, it is important to consider the possibility that the pathologic abnormality may be spontaneous CCF rather than conjunctivitis. Ophthalmologist may be the first physician to encounter a patient with clinical manifestations of $\mathrm{CCF}$ and should raise awareness of the clinical features of spontaneous CCF to avoid misdiagnosis.

\section{References}

1. Barrow DL, Spector RH, Braun IF, Landman JA, Tindall SC, et al. (1985) Classification and treatment of spontaneous carotid-cavernous sinus fistulas. J Neurosurg 62: 248256. [Crossref]
2. Liu HM, Wang YH, Chen YF, Cheng JS, Yip PK, et al. (2001) Long-term clinical outcome of spontaneous carotid cavernous sinus fistulae supplied by dural branches of the internal carotid artery. Neuroradiology 43: 1007-1014. [Crossref]

3. Grumann AJ, Boivin-Faure L, Chapot R, Adenis JP, Robert PY (2012) Ophthalmologic outcome of direct and indirect carotid cavernous fistulas. Int Ophthalmol 32: 153-159. [Crossref]

4. Chaudhry IA, Elkhamry SM, Al-Rashed W, Bosley TM (2009) Carotid cavernous fistula: ophthalmological implications. Middle East Afr J Ophthalmol 16: 57-63. [Crossref]

5. Feiner L, Bennett J, Volpe NJ (2003) Cavernous sinus fistulas: carotid cavernous fistulas and dural arteriovenous malformations. Curr Neurol Neurosci Rep 3: 415-420. [Crossref]

6. Miller NR (2012) Dural carotid-cavernous fistulas: epidemiology, clinical presentation, and management. Neurosurg Clin North Am 23: 179-192. [Crossref]

Copyright: (C2018 Xia Y. This is an open-access article distributed under the terms of the Creative Commons Attribution License, which permits unrestricted use, distribution, and reproduction in any medium, provided the original author and source are credited. 